\title{
Tendinitis crónica no insercional del Aquiles, una nueva opción para su manejo
}

\author{
Chronic Achilles tendinopathy, a new option for treatment
}

\author{
Ana Cristina King Martínez, ${ }^{*}$ Luciano Torres Sánchez, ${ }^{\ddagger}$ Alberto Cuéllar Avaroma ${ }^{\S}$
}

Citar como: King MAC, Torres SL, Cuéllar AA. Tendinitis crónica no insercional del Aquiles, una nueva opción para su manejo. An Med (Mex). 2021; 66 (1): 57-62. https://dx.doi.org/10.35366/99491

\section{RESUMEN}

La tendinitis crónica no insercional del Aquiles consiste en la tríada clínica de dolor, edema y dificultad para la marcha. El origen de esta patología es debido a una combinación de uso excesivo que conduce a microtraumatismos repetitivos, mala vascularización del tejido, desequilibrios mecánicos de la extremidad, predisposición genética y una variedad de factores metabólicos. Inicialmente, los pacientes son manejados de manera conservadora corrigiendo algunos de los factores etiológicos subyacentes, seguidos de modificación de la actividad, medicamentos y programas de rehabilitación. La cirugía se considera una opción aceptable para los pacientes que no responden al tratamiento inicial. Se presenta el caso de una mujer de 49 años, quien inicia con tumoración dolorosa a nivel del cuerpo del tendón de Aquiles izquierdo de un año de evolución, que llegó hasta incapacitarla para la marcha. La paciente fue inicialmente valorada por un médico general, quien la refirió a un centro oncológico por sospecha de tumoración en el tendón de Aquiles, posteriormente fue enviada a nuestro servicio. Nosotros integramos un diagnóstico de tendinitis no insercional crónica del Aquiles. Se inicia manejo conservador, pero al ser insuficiente, tras cuatro meses se decide un tratamiento quirúrgico. Se realiza una tenoscopia del Aquiles con desbridamiento, además de una artroscopia posterior del tobillo con una transferencia del flexor largo del hallux. A un año de seguimiento de la cirugía, la paciente presenta una marcha independiente, no dolorosa con retorno a sus actividades diarias sin complicaciones.

\section{ABSTRACT}

The chronic insertional Achilles tendinopathy sin consistent with: pain, swelling, and difficulty to walk. The origin of this pathology is due to a combination of excess of stress forces that lead to repetitive microtrauma, suboptimal tissue vascularization, muscular unbalance of the extremity, genetic predisposition and metabolic factors. Initial the patients receive a non-surgical approach that tries to correct some ethological factors, followed by modifications in the physical activities, current medical treatments, and a formal rehabilitation program. The surgery is consider an option only in patients who do not respond to initial treatment. We present a case of a 49 year old female patient, who developed a painful growth in the left Achilles tendon body. After a year her walking was impaired. A general physician, whom with the suspicion of a tumor sends her to an oncology center, initially assessed the patient. Afterwards she was send to our service, where we made a diagnostic of chronic non-insertional Achilles tendinopathy. We initiated a non-surgical approach, but after four months a surgical treatment was decided. We performed a tendinoscopy of the Achilles, with debridement of the tendon associated with a posterior ankle arthroscopy and a flexor halluces longus transfer. We followed the patient for a year after which she had an independent and non-painful gait, she had returned to her daily activities with out any complications.

\footnotetext{
* Médico Ortopedista, Jefatura de la División de Ortopedia, Hospital General «Dr. Manuel Gea González». México.

₹ Médico residente de Ortopedia, División de Ortopedia, Hospital General «Dr. Manuel Gea González». México.

$\S$ Médico Ortopedista, Hospital Médica Sur. México.
}

Correspondencia: Dr. Alberto Cuéllar Avaroma

Puente de Piedra Núm. 150, torre 2, consultorio 118, Col. Toriello Guerra, 14050, Tlalpan, Ciudad de México. Tel: 55 5666-0808

E-mail: alberto.cuellar@kingortopedia.com

Abreviaturas:

$\mathrm{FHL}=$ Flexor hallux longus 
Palabras clave: Tendón de Aquiles, transferencia, tratamiento.

\section{INTRODUCCIÓN}

La tendinopatía de Aquiles es una afección clínica caracterizada por dolor e inflamación en y alrededor del tendón, principalmente debido al uso excesivo, pero que a menudo se presenta en pacientes de mediana edad con sobrepeso sin antecedentes de aumento en la actividad física. ${ }^{1}$

Según el tiempo de evolución de los síntomas puede clasificarse en aguda, y crónica si es mayor de seis semanas. $^{2}$

La tendinitis de Aquiles primero debe diferenciarse según la ubicación anatómica; se puede subdividir en insercional (que surge en la unión tendón-Aquiles) o no insercional (ubicada $2-6 \mathrm{~cm}$ proximal a esta inserción de Aquiles).

La incidencia de la tendinopatía de Aquiles en corredores de alto nivel se ha estimado en alrededor de 7 a 9\%; sin embargo, no sólo ocurre en deportistas, se estima que hasta un tercio de las personas afectadas no son deportistas. ${ }^{3}$ En población general tiene una incidencia de 1.85 por 1,000 habitantes. ${ }^{4}$ Siendo esto quizás subvaluado debido a deficiencias para su diagnóstico, como en este caso que la paciente fue enviada a un centro especializado en patologías oncológicas debido al aumento de volumen de su tendón. Tampoco contamos con registros epidemiológicos suficientes sobre la patología.

Existen factores que se han identificado como predisponentes, éstos se pueden clasificar como intrínsecos (es decir, edad, sexo, índice de masa corporal, anomalías biomecánicas, mala alineación del pie, disfunción del gastrocnemio-sóleo, inestabilidad del tobillo, etc.) ${ }^{3}$ y extrínsecos (es decir, uso de esteroides, fluoroquinolonas, entrenamiento inadecuado, factores ambientales, calzado, etc.). ${ }^{5}$

Los factores como la degeneración intratendinosa (tendinitis), la neovascularización y el proceso inflamatorio neurogénico tienen el papel más importante en la presencia de dolor en los pacientes de acuerdo con Lohrer. ${ }^{6}$

\section{CASO CLÍNICO}

Paciente femenino de 49 años, nacionalidad mexicana, ocupación hogar, con antecedente de hiper-
Keywords: Achilles tendon, tendon transfer, treatment.

tensión arterial sistémica de 10 años de evolución con tratamiento médico. Inicia con padecimiento actual un año previo a su ingreso a esta unidad, con tumoración a nivel del cuerpo del tendón de Aquiles izquierdo con dolor a la palpación. El dolor fue en aumento hasta incapacitar la marcha independiente.

Es valorada inicialmente por médico general, quien con un diagnóstico de tumoración en el tendón de Aquiles la refiere al Instituto Nacional de Cancerología. En dicha institución realizan protocolo de estudio. Al descartar patología maligna envían a la paciente a la División de Ortopedia del Hospital General «Dr. Manuel Gea González».

En nuestra valoración inicial encontramos a la exploración física dirigida, paciente neurológicamente íntegra con marcha bipodálica claudicante a expensas de miembro pélvico izquierdo de no más de cuatro pasos. La paciente acude en silla de ruedas. Se observa aumento de volumen a nivel del cuerpo del tendón de Aquiles, se palpa tumoración dura (Figura 1), dolorosa, no adherida a campos profundos, la cual se desplaza con la flexoextensión del tobillo. No encontramos cambios en la coloración, ni temperatura, arcos de movilidad de cadera y rodilla completos. A la exploración del tobillo observamos disminución de flexión plantar a $10^{\circ}$ y dorsiflexión de $20^{\circ}$, ambos movimientos eran dolorosos. La fuerza por grupos musculares con incapacidad para pararse de puntas (Figura 2) a nivel de tríceps sural 3/5 escala Daniels, tibial anterior 5/5. La sensibilidad distal por dermatomos L1-S2 conservada. Los pulsos pedios y tibial posterior presentes de buena intensidad, llenado capilar distal dos segundos. Maniobras especiales para tendón de Aquiles Thompson presente, signo del hachazo negativo. Prueba del Royal London Hospital positiva (Figura 3). Se realiza escala AOFAS (por sus siglas en inglés de American Orthopaedic Foot and Ankle Society) de tobillo y retropié con puntuación de $23 / 100$.

Resonancia magnética simple del tendón de Aquiles en la que se observa aumento de diámetro del tendón de Aquiles e hiperintensidad en grasa de Kager, aumento de volumen en la mayor parte del trayecto del tendón, con aumento de su diámetro e imágenes compatibles con rupturas focales. No hay presencia de nódulos (Figura 4). 
Tras la exploración física y el análisis de su estudio se integra el diagnóstico de tenosinovitis no insercional crónica del Aquiles.

Se inicia manejo no quirúrgico con bota tipo Walker y terapia física donde se realiza especial énfasis en ejercicios de elasticidad del tendón de Aquiles. Se indica el uso de talonera dentro de la bota Walker iniciando con una flexión plantar de $10^{\circ}$. La deambulación se indicó sin apoyo de la extremidad con ayuda de muletas. A las dos semanas se cita nuevamente y se retira la talonera llevando el tobillo a $90^{\circ}$. Se permite el apoyo a tolerancia y se continúa el uso de la bota Walker. La paciente acude hasta tres meses después con mejoría importante de la sintomatología dolorosa, ya sin uso de bota Walker; sin embargo, aún no puede realizar marcha independiente. Continúa con tumoración a nivel del tendón de Aquiles, pero ésta ha disminuido en $50 \%$ su tamaño. Aún con dolor a la palpación de la zona. La paciente no puede pararse de puntas por insuficiencia del tendón de Aquiles.

Se le propone manejo quirúrgico consistente en artroscopia posterior de tobillo izquierdo con transposición del tendón flexor hallux longus (FHL) al calcáneo así como tenoscopia del Aquiles.

Se realiza la cirugía programada, se efectúa inicialmente la tenoscopia del Aquiles encontrando importante fibrosis entre el tendón y el peritendón, la cual se libera. A continuación se realiza artroscopia posterior de tobillo haciendo una resección parcial de la grasa de Kager que se encontraba inflamada,

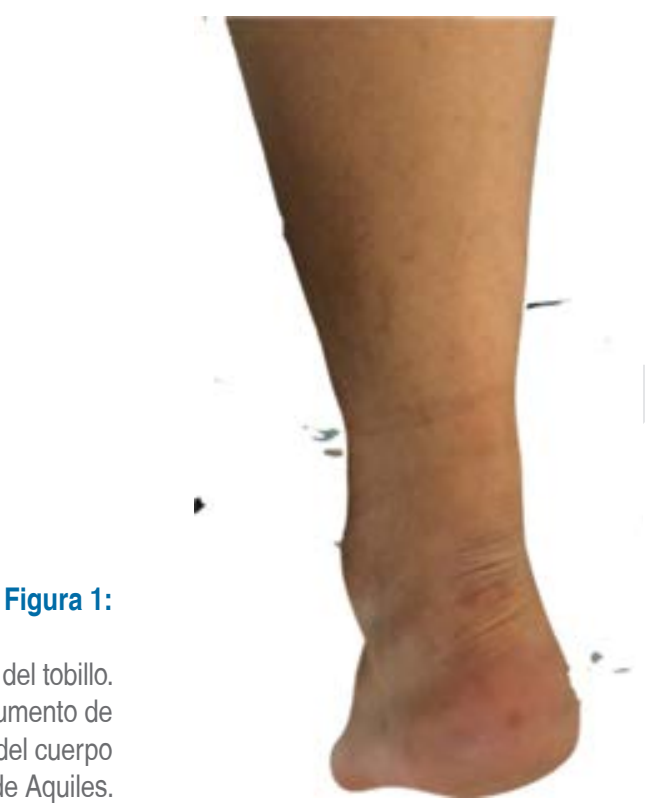

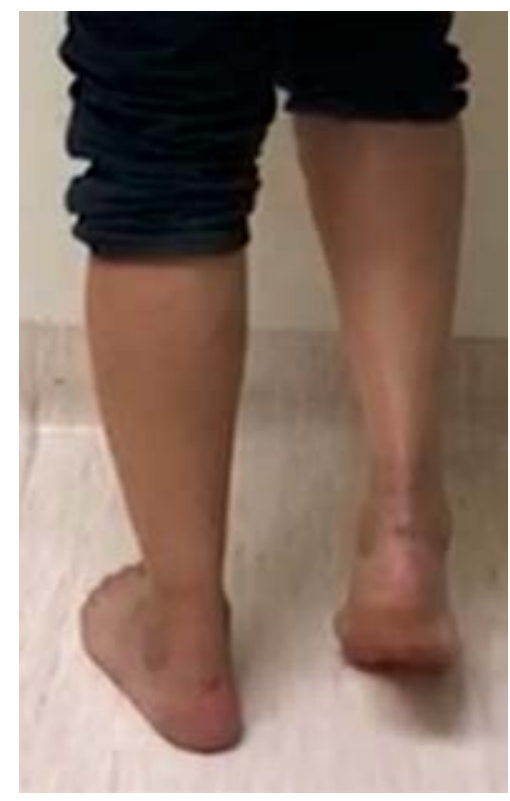

Figura 2:

Se observa paciente en bipedestación, con incapacidad para pararse de puntas en tobillo izquierdo.

se localiza el tendón flexor largo del hallux, se realiza tenotomía con ayuda de radiofrecuencia (VULCAN Smith \& Nephew). Se procede a extraer el FHL en el calcáneo. Se perfora con clavo guía y sobre de éste con broca canulada de $6 \mathrm{~mm}$ se hace un túnel de unos $25 \mathrm{~mm}$, se pasa la guía y se deja una sutura en asa para la tracción del injerto. Se introduce el injerto en el túnel y se tracciona por vía plantar para tener una adecuada tensión; se fija con un tornillo interferencial biocompuesto $7 \times 25 \mathrm{~mm}$ (BIORCI Smith \& Nephew). Posterior al evento quirúrgico la paciente se inmoviliza con una bota Walker con $10^{\circ}$ de flexión plantar. A las dos semanas, el equino se redujo y el tobillo se colocó en una posición neutral en una bota Walker. Entonces se inició la carga de peso tolerada con muletas. A las cuatro semanas después de la operación se retiró la bota Walker y se alentó la libre movilidad del tobillo. Se continuó con muletas hasta que la paciente se sintió cómoda para movilizarse sin ayuda. La fisioterapia formal se inició a las cuatro semanas con ejercicios que aumentaron progresivamente la fuerza y el rango de movimiento de la articulación del tobillo.

Se dio seguimiento clínico a la paciente de un año a partir del procedimiento quirúrgico, sin dolor, con marcha independiente llevando a cabo sus actividades diarias sin limitaciones, podía realizar marcha de punta y talón sin problemas, podía levantarse de puntas en apoyo monopodálico sobre el pie operado. La paciente no hacía deporte previo a la cirugía y no lo había iniciado después. Se realiza escala AOFAS 
de tobillo y retropié observando una puntuación de 90/100.

Se encontraba muy satisfecha con el resultado de la cirugía.

\section{DISCUSIÓN}

Se ha demostrado que los factores metabólicos, dentro de los que se incluye la hipertensión (nuestra paciente era hipertensa de larga evolución), la hiperlipidemia y la obesidad predisponen a la tendinopatía de Aquiles y a la rotura. Se ha propuesto que todos estos factores actúan a través de una vía común en la disminución de la vascularización del tejido y, posteriormente, disminución del potencial de curación. Se ha demostrado que tanto la hipertensión como la obesidad disminuyen los niveles de óxido nítrico, una vía utilizada para establecer la dilatación vascular y aumentar el flujo sanguíneo en las zonas lesionadas. ${ }^{7}$

La esencia de la tendinopatía de Aquiles es una respuesta de cicatrización fallida, con proliferación desordenada de tenocitos, alguna evidencia de degeneración en las células de los tendones y rotura de las fibras de colágeno, y el subsecuente aumento de la matriz no colágena, lo que lleva a un tendón mecánicamente menos estable que es más susceptible al daño.

El diagnóstico de la tendinopatía corporal de Aquiles se basa principalmente en una historia y un examen clínico detallado, este último sigue siendo la mejor herramienta de diagnóstico. Se puede llegar a

Figura 3:

Prueba del Royal London Hospital: una vez que el examinador ha provocado dolor local a la palpación del tendón con el tobillo en posición neutra, se le pide a la paciente que realice una dorsiflexión activa del tobillo y una flexión plantar activa. Dorsiflexión máxima y en flexión plantar máxima, se vuelve a palpar la porción del tendón que originalmente se encontró sensible. observar y palpar un aumento de volumen a nivel de 2-6 cm proximal a la inserción en el calcáneo. Se debe evaluar la marcha y pedirle al paciente que se pare de puntas, esto le ocasiona dolor o incluso incapacidad para realizarlo. Se puede utilizar la escala AOFAS de tobillo y retropié para evaluar la funcionalidad, dolor y alineación del tobillo del paciente, ayudando de manera objetiva a cuantificar la evolución así como la satisfacción del paciente con el tratamiento.

Se deben realizar estudios de imagen para complementar el diagnóstico, puede ser un ultrasonido; sin embargo, éste cuenta con la limitante de ser operado dependiente, por lo que en nuestro medio lo que más se utiliza es la resonancia magnética donde se pueden observar las estructuras anatómicas y tejidos blandos y los cambios directos e indirectos en la degeneración del tendón.

En el caso de la tendinopatía de Aquiles se debe iniciar un tratamiento conservador, la literatura nos refiere que debe ser de al menos seis meses antes de recomendar la cirugía. ${ }^{5}$ Se debe intentar abordar y posiblemente corregir algunos de los factores etiológicos subyacentes, seguido de un enfoque multimodal, que incluya modificación de la actividad, medicamentos y programas de estiramiento/fortalecimiento. Hay investigaciones que respaldan 12 semanas de ejercicio excéntrico para reducir el dolor y la neovascularización, aunque la evidencia es poco concluyente. ${ }^{8}$ La evidencia que respalda el uso de otras modalidades conservadoras es limitada, incluidos medicamentos antiinflamatorios, ultrasonido, terapia de ondas de choque, inyecciones de corticosteroides, terapia plasma rico en plaquetas. ${ }^{9,10}$

Aproximadamente de 24 a $45 \%$ de los pacientes fracasan en el tratamiento conservador después de seis meses. ${ }^{11}$ Las contraindicaciones para la cirugía incluyen insuficiencia arterial, infección cutánea activa, compromiso de los tejidos blandos y determinadas comorbilidades médicas que hacen de un paciente un candidato quirúrgico de alto riesgo.

El propósito del tratamiento quirúrgico de pacientes con tendinopatía de Aquiles es reducir los síntomas y mejorar la función en individuos activos que no han respondido al tratamiento conservador. Aunque se ha informado que los resultados de la cirugía abierta son satisfactorios, se han producido hasta $11 \%$ de complicaciones, $54 \%$ relacionadas con la herida quirúrgica. ${ }^{12}$

De acuerdo con los abordajes terapéuticos existen varios caminos para reducir el dolor y mejorar las condiciones del tendón: ${ }^{6,13}$ 

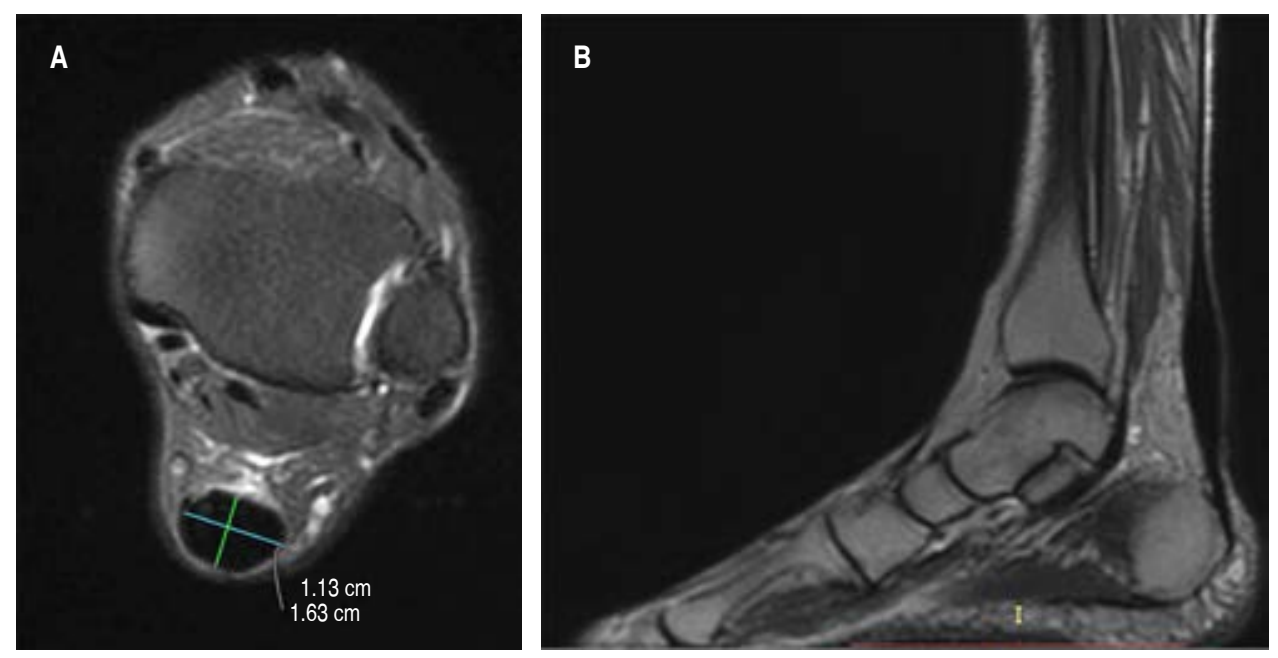

Figura 4:

Resonancia magnética simple en T2. Corte axial y sagital. A) Se observa aumento de diámetro del tendón de Aquiles e hiperintensidad en grasa de Kager. B) Aumento de volumen en cuerpo del tendón de Aquiles.

1. Retiro del tejido anormal del tendón y paratendón.

2. Activar la regeneración tendinosa a través de la escarificación de la misma.

3. Disrupción vascular.

4. Liberación de los gastrocnemios para disminuir la tensión sobre el tendón de Aquiles.

5. Si la calidad del tendón es mala en casos de tendinitis crónicas, se sugiere la transferencia del FHL o bien cuando el desbridamiento realizado al tendón de Aquiles es igual o mayor a 50\% del mismo.

Nuestra paciente al presentar una patología crónica que se acompañaba ya de lesiones intratendinosas, así como insuficiencia muscular importante, consideramos realizar el desbridamiento artroscópico más la transferencia del FHL como tratamiento. La transferencia del tendón FHL vía artroscópica es una alternativa válida para tratar la tendinopatía de Aquiles mejorando la calidad de vida con menor riesgo de complicaciones postquirúrgicas, ${ }^{12,14,15}$ la cual está indicada en pacientes que no sean deportistas de alto rendimiento o en pacientes con baja demanda funcional como es el caso de nuestra paciente. ${ }^{16}$

Sin embargo, hoy en día, debido a que es una técnica relativamente reciente, no se cuenta con estudios suficientes a largo plazo.

Realizar el procedimiento de transferencia por vía artroscópica ha demostrado los mismos resultados funcionales con una importante disminución en las complicaciones que presentan los pacientes, 5.3\% de complicaciones vs. $11 \%$ en técnicas abiertas tradicionales. El procedimiento artroscópico también se asocia con $91 \%$ de satisfacción del paciente vs. $79 \%$ en los procedimientos abiertos según Lohrer. ${ }^{6}$
Nuestro caso puede ser comparado con los resultados obtenidos por Lohrer, donde encontramos pocas complicaciones, una adecuada satisfacción del paciente posterior al evento quirúrgico y una recuperación y reintegración a sus actividades diarias en un corto tiempo.

Podemos comparar también los resultados obtenidos en la serie de Baumfeld, ${ }^{17}$ nuestra paciente se encuentra en el rango de edad reportado por ellos, el tiempo quirúrgico en su serie fue similar al nuestro, no tuvimos complicaciones de las heridas al igual que lo descrito en la serie. Nosotros dimos seguimiento de un año a la paciente que fue igual que el caso más largo de seguimiento que ellos presentan en su serie. Los resultados clínicos que obtuvimos con nuestra paciente son comparables con los reportados en la serie, logrando una adecuada movilidad del tobillo, buena fuerza al pararse en un pie y pararse de puntas en un solo pie.

De acuerdo con nuestros resultados contrastados con lo descrito en la bibliografía podemos reportar un resultado satisfactorio en nuestra paciente.

\section{CONCLUSIÓN}

La tendinitis crónica no insercional del Aquiles es una patología poco común, mal diagnosticada que condiciona discapacidad importante al paciente. El diagnóstico se basa principalmente en una historia clínica minuciosa y exploración física; sin embargo, se utilizan estudios complementarios como la resonancia magnética para apoyarlo. El tratamiento inicial es conservador; sin embargo, $25 \%$ de los pacientes que persisten con síntomas pueden requerir tratamiento quirúrgico. El propósito del trata- 
An Med (Mex) 2021; 66 (1): 57-62

miento quirúrgico de pacientes con tendinopatía de Aquiles es reducir los síntomas y mejorar la función del tendón en individuos activos que no han respondido al tratamiento conservador. Aunque se ha informado que los resultados de la cirugía abierta son satisfactorios, se han producido hasta $11 \%$ de complicaciones, por lo que se han buscado opciones menos invasivas.

Es por esto que se propone la transferencia del tendón FHL vía artroscópica, siendo una alternativa válida para tratar tendinopatía de Aquiles mejorando la calidad de vida con menor riesgo de complicaciones postquirúrgicas.

\section{REFERENCIAS}

1. Maffulli N, Khan KM, Puddu G. Overuse tendon conditions: time to change a confusing terminology. Arthroscopy. 1998; 14 (8): 840-843.

2. Wilcox DK, Bohay DR, Anderson JG. Treatment of chronic Achilles tendon disorders with flexor hallucis longus tendon transfer/augmentation. Foot Ankle Int. 2000; 21 (12): 10041010.

3. Longo UG, Ronga M, Maffulli N. Achilles tendinopathy. Sports Med Arthrosc Rev. 2009; 17 (2): 112-126.

4. de Jonge S, van den Berg C, de Vos RJ, van der Heide HJ, Weir A, Verhaar JA et al. Incidence of midportion Achilles tendinopathy in the general population. Br J Sports Med. 2011; 45 (13): 1026-1028.

5. Singh A, Calafi A, Diefenbach C, Kreulen C, Giza E. Noninsertional tendinopathy of the achilles. Foot Ankle Clin. 2017; 22 (4): 745-760.

6. Lohrer H, David S, Nauck T. Surgical treatment for Achilles tendinopathy - a systematic review. BMC Musculoskelet Disord. 2016; 17: 207.

7. Williams IL, Wheatcroft SB, Shah AM, Kearney MT. Obesity, atherosclerosis and the vascular endothelium: mechanisms of reduced nitric oxide bioavailability in obese humans. Int $\mathrm{J}$ Obes Relat Metab Disord. 2002; 26 (6): 754-764.

8. Zwiers R, Wiegerinck JI, van Dijk CN. Treatment of midportion Achilles tendinopathy: an evidence-based overview. Knee Surg Sports Traumatol Arthrosc. 2016; 24 (7): 2103-2111.

9. Andres BM, Murrell GA. Treatment of tendinopathy: what works, what does not, and what is on the horizon. Clin Orthop Relat Res. 2008; 466 (7): 1539-1554.

10. Rompe JD, Furia JP, Maffulli N. Mid-portion Achilles tendinopathy--current options for treatment. Disabil Rehabil. 2008; 30 (20-22): 1666-1676.

11. Paavola M, Kannus P, Paakkala T, Pasanen M, Jarvinen M. Long-term prognosis of patients with achilles tendinopathy. An observational 8-year follow-up study. Am J Sports Med. 2000; 28 (5): 634-642.

12. Paavola M, Orava S, Leppilahti J, Kannus P, Jarvinen M. Chronic Achilles tendon overuse injury: complications after surgical treatment. An analysis of 432 consecutive patients. Am J Sports Med. 2000; 28 (1): 77-82.

13. Schmidtberg B, Johnson JD, Kia C, Baldino JB, Obopilwe E, Cote MP et al. Flexor hallucis longus transfer improves achilles tendon load to failure in surgery for non-insertional tendinopathy: a biomechanical study. J Bone Joint Surg Am. 2019; 101 (16): 1505-1512.

14. Syed TA, Perera A. Endoscopic management of chronic Achilles tendon rupture. Foot Ankle Clin. 2019; 24 (3): 459470.

15. Baltes TPA, Zwiers R, Wiegerinck JI, van Dijk CN. Surgical treatment for midportion Achilles tendinopathy: a systematic review. Knee Surg Sports Traumatol Arthrosc. 2017; 25 (6): 1817-1838.

16. Vega J, Vilá J, Batista J, Malagelada F, Dalmau-Pastor M. Endoscopic flexor hallucis longus transfer for chronic noninsertional Achilles tendon rupture. Foot Ankle Int. 2018; 39 (12): 1464-1472.

17. Baumfeld D, Baumfeld T, Figueiredo AR, de Araujo Junior LF, Macedo B, Silva TAA et al. Endoscopic flexor halluces longus transfer for chronic achilles tendon rupture - technique description and early post-operative results. Muscles Ligaments Tendons J. 2017; 7 (2): 341-346. 